This is a preprint of: "The cyclicity of the period annulus of a reversible quadratic system", Changjian Liu, Chengzhi Li, Jaume Llibre, Proc. Roy. Soc. Edinburgh Sect. A, 1-10, 2021.

\title{
THE CYCLICITY OF THE PERIOD ANNULUS OF A REVERSIBLE QUADRATIC SYSTEM
}

\author{
CHANGJIAN LIU ${ }^{1}$, CHENGZHI LI² AND JAUME LLIBRE ${ }^{3}$
}

\begin{abstract}
We prove that perturbing the periodic annulus of the reversible quadratic polynomial differential system $\dot{x}=y+a x^{2}$, $\dot{y}=-x$ with $a \neq 0$ inside the class of all quadratic polynomial differential systems we can obtain at most two limit cycle, including their multiplicities. Since the first integral of the unperturbed system contains an exponential function, the traditional methods can not be applied, except in [6] a computer-assisted method was used. In this paper we provide a method for studying the problem. This is also the first purely mathematical proof of the conjecture formulated by F. Dumortier and R. Roussarie in [5] for $q \leq 2$. The method may be used in other problems.
\end{abstract}

\section{IntRoduction AND STATEMENT OF THE MAIN RESUlts}

We recall that a center of a planar differential system is a singular point $p$ of the system having a neighborhood filled up of periodic orbits with the unique exception of the point $p$. The period annulus of a center is the maximal region filled up with the periodic orbits surrounding the center.

There is a big program whose objective is to find the exact upper bound for the number of limit cycles that can bifurcate from the periodic orbits of the period annuli of the quadratic polynomial differential systems under quadratic perturbations, see for instance the second part of the book of C. Christhopher and C. Li [3]. This upper bound is called the cyclicity of the period annulus. This program started with V. I. Arnold [1, 2] and has produced more than one hundred articles, see for instance the references of [3].

Here we contribute to this program determining this upper bound for the period annulus of the center of the quadratic polynomial differential

2010 Mathematics Subject Classification. Primary 34C07, 34C08, 37G15.

Key words and phrases. Perturbation of quadratic reversible center, Abelian integral, limit cycle. 
systems

$$
\dot{X}=Y+a X^{2}, \quad \dot{Y}=-X,
$$

with $a \neq 0$. We note that to study the cyclicity of the period annulus of system (1) is equivalent to study the cyclicity of the period annulus of the system

$$
\dot{x}=y+4 x^{2}, \quad \dot{y}=-x .
$$

Indeed, doing the change of variables $(X, Y) \rightarrow(x, y)$ where $X=4 x / a$ and $Y=4 y / a$ system (1) becomes system (2).

System (2) has the first integral

$$
H(x, y)=e^{8 y}\left(4 x^{2}+y-\frac{1}{8}\right)
$$

and the corresponding integrating factor $R(y)=8 e^{8 y}$.

The phase portrait of system (2) in the Poincaré disc is shown in Figure 1. This phase portrait has a unique finite singular point, the

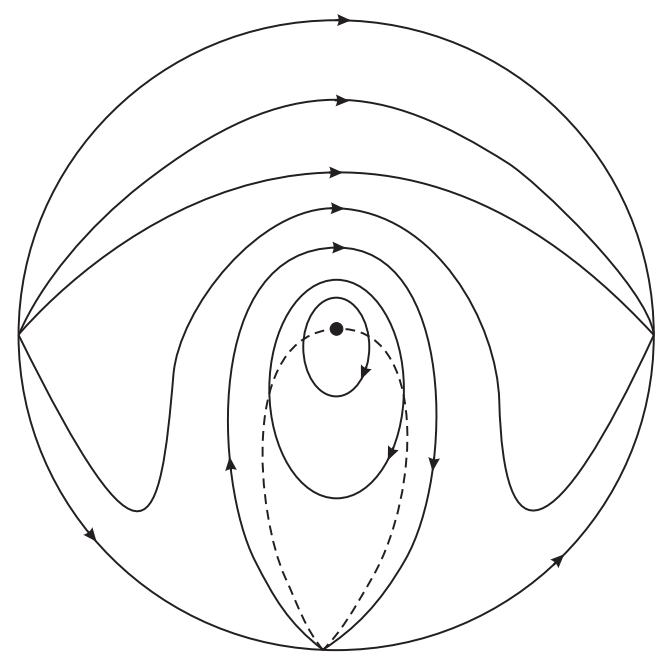

Figure 1. The phase portrait of system (2) in the Poincaré disc, with the parabola $y=-4 x^{2}+1 / 8$ at the boundary of the period annulus.

center localized at the origin of coordinates. It has two pairs of infinite singular points localized at the endpoints of the $x$ and $y$ axes. At the endpoint of the positive $x$-half-axis there is a hyperbolic stable node, at the endpoint of the negative $x$-half-axis there is a hyperbolic unstable node, at the endpoints of the $y$-axis there is a nilpotent saddle, having a hyperbolic sector at the endpoint of the positive $y$-half-axis and three hyperbolic sectors at the endpoint of the negative $y$-axis. 
For the definitions of first integral and integrating factor see Chapter 8, for the definition of the Poincaré disc see Chapter 5, and for the definitions of hyperbolic and nilpotent singular points see Chapters 2 and 3 respectively of [4]. The boundary of the period annulus of the center of system (2) localized at the origin of coordinates is the parabola $y=-4 x^{2}+1 / 8$. Then the period annulus can be expressed by $\left\{\gamma_{h}: h \in(-1 / 8,0)\right\}$, where $\gamma_{h}$ is the periodic orbit

$$
H(x, y)=e^{8 y}\left(4 x^{2}+y-\frac{1}{8}\right)=h .
$$

In what follows we will say simply quadratic system instead of quadratic polynomial differential system. It is known, see for instance [7], that any reversible quadratic system can be written in the complex form $\dot{z}=-i z+a z^{2}+2|z|^{2}+b \bar{z}^{2}$ where $z=x+i y$, or in the real form

$$
\dot{x}=y+(a+b+2) x^{2}-(a+b-2) y^{2}, \quad \dot{y}=-x+2(a-b) x y,
$$

where $a$ and $b$ are real parameters. When $a=b=1$ the reversible quadratic system (4) becomes system (2).

Our main result is the following one.

Theorem 1. The cyclicity of the period annulus of system (2) under quadratic perturbations is two.

Theorem 1 is proved in next section.

Remark 2. Note that system (8) of [5] is just our system (1) with $a=-1$, hence the Abelian integrals $I_{1}(h), I_{3}(h), I_{5}(h)$ for $h \in(-1 / 8,0)$ in Lemma 4 are equivalent to $J_{1}(h), J_{3}(h), J_{5}(h)$ for $h \in(0,1 / 2)$ in [5]. $F$. Dumortier and $R$. Roussarie formulated a conjecture on page 726 of [5], that $\left\{J_{1}^{\prime}(h), J_{3}^{\prime}(h), \ldots J_{2 q+1}^{\prime}(h)\right\}$ forms a strict Chebyshev system for $h \in(0,1 / 2)$ and for any integer $q \geq 0$. This conjecture is obviously true for $q=0$. We give a positive answer to this conjecture for $q=1$ in Lemma 6 and for $q=2$ in Lemmas 7-9, by using purely mathematical method. Note also that J. Figuerasa, W. Tucker and J. Villadelprat in [6] gave a proof of this conjecture for $q \leq 2$ by using theoretical analysis and computations by computer, that are based on computerassisted techniques. For example, the computations for the proof of a lemma take six and a half hours on a desktop computer with a $2.8 \mathrm{GHz}$ CPU, see Remark 4.11 of [6]. 


\section{Proof of Theorem 1}

We first state a result by I. D. Iliev, see statement (ii). (3) with $a=b=1$ in Theorem 2 of [7].

Theorem 3. The exact upper bound for the number of limit cycles produced by the period annulus of the reversible quadratic system (2) under quadratic perturbations is equal to the maximal number of zeros in the interval $(-1 / 8,0)$ counting multiplicities of the function

$$
M(h)=\iint_{H(x, y)<h} 8 e^{8 y}\left(\mu_{1}+\mu_{2} y+\mu_{3} y^{2}\right) d x d y,
$$

where $\mu_{1}, \mu_{2}$ and $\mu_{3}$ are arbitrary constants, and $\mu_{1}^{2}+\mu_{2}^{2}+\mu_{3}^{2} \neq 0$.

Lemma 4. For $h \in(-1 / 8,0)$ the function (4) can be expressed as

$$
M(h)=\alpha_{1} I_{1}(h)+\alpha_{2} I_{3}(h)+\alpha_{3} I_{5}(h),
$$

where $\alpha_{1}, \alpha_{2}$ and $\alpha_{3}$ are arbitrary constants, $\alpha_{1}^{2}+\alpha_{2}^{2}+\alpha_{3}^{2} \neq 0$, and

$$
I_{k}(h)=\int_{\gamma_{h}} 8 e^{8 y} x^{k} d y, \quad k=1,3,5 .
$$

Proof. It is obviously that (4) can be expressed as

$$
\int_{\gamma_{h}} e^{8 y}\left(\bar{\mu}_{1}+\bar{\mu}_{2} y+\bar{\mu}_{3} y^{2}\right) d x
$$

where $\bar{\mu}_{1}, \bar{\mu}_{2}$ and $\bar{\mu}_{3}$ are arbitrary constants and $\bar{\mu}_{1}^{2}+\bar{\mu}_{2}^{2}+\bar{\mu}_{3}^{2} \neq 0$. Hence, we only need to prove that each $\int_{\gamma_{h}} e^{8 y} y^{k} d x$ (for $\left.k=0,1,2\right)$ can be expressed as a linear combination of $I_{1}(h), I_{3}(h)$ and $I_{5}(h)$.

First, using integration by parts we have

$$
\int_{\gamma_{h}} e^{8 y} d x=-I_{1}(h)
$$

Next, by using (3) and integration by parts we have

$$
\int_{\gamma_{h}} e^{8 y} y d x=\int_{\gamma_{h}}\left[h+e^{8 y}\left(\frac{1}{8}-4 x^{2}\right)\right] d x=-\frac{1}{8} I_{1}(h)+\frac{4}{3} I_{3}(h) .
$$

Finally, by using integration by parts we have

$$
\int_{\gamma_{h}} e^{8 y} y^{2} d x=-2 \int_{\gamma_{h}} x y e^{8 y} d y-8 \int_{\gamma_{h}} x y^{2} e^{8 y} d y=-2 K_{1}(h)-8 K_{2}(h) .
$$

From (2) we have

$$
x d x+\left(y+4 x^{2}\right) d y=0
$$


Multiplying (8) by $x e^{8 y}$ we have

$$
K_{1}(h)=-\frac{1}{2} I_{3}(h)-\oint_{\gamma_{h}} x^{2} e^{8 y} d x=-\frac{1}{6} I_{3}(h) .
$$

Multiplying (8) by $x y e^{8 y}$ we have

$$
\begin{aligned}
K_{2}(h) & =-4 \oint_{\gamma_{h}} x^{3} y e^{8 y} d y-\oint_{\gamma_{h}} x^{2} y e^{8 y} d x \\
& =-4 \oint_{\gamma_{h}} x^{3}\left[d\left(\frac{1}{8} y e^{8 y}\right)-\frac{1}{8} e^{8 y} d y\right]-\oint_{\gamma_{h}} x^{2} y e^{8 y} d x \\
& =\frac{1}{2} \oint_{\gamma_{h}} x^{2} y e^{8 y} d x+\frac{1}{16} I_{3}(h) .
\end{aligned}
$$

Using (3), we change the first integral above (neglecting the fact $\frac{1}{2}$ ) to

$$
\begin{aligned}
& \oint_{\gamma_{h}} x^{2} y e^{8 y} d x=\oint_{\gamma_{h}} x^{2}\left[h+\left(\frac{1}{8}-4 x^{2}\right) e^{8 y}\right] d x \\
& =\oint_{\gamma_{h}} x^{2}\left(\frac{1}{8}-4 x^{2}\right) e^{8 y} d x=\frac{1}{8} \oint_{\gamma_{h}} x^{2} e^{8 y} d x-4 \oint_{\gamma_{h}} x^{4} e^{8 y} d x \\
& =-\frac{1}{24} I_{3}(h)+\frac{4}{5} I_{5}(h) .
\end{aligned}
$$

Lemma 5. For $h \in(-1 / 8,0)$ the function $M(h)$ in (5) satisfies

$$
M^{\prime}(h)=\alpha_{1} I_{1}^{\prime}(h)+\alpha_{2} I_{3}^{\prime}(h)+\alpha_{3} I_{5}^{\prime}(h),
$$

where

$$
I_{k}^{\prime}(h)=\int_{\gamma_{h}} k x^{k-2} d y, \quad k=1,3,5
$$

Proof. It is easy to check by (3) that along $\gamma_{h}$ one has

$$
\frac{\partial x}{\partial h}=\frac{1}{8 e^{8 y} x}
$$

thus

$$
I_{k}^{\prime}(h)=\int_{\gamma_{h}} 8 e^{8 y} k x^{k-1} \frac{\partial x}{\partial h} d y=\int_{\gamma_{h}} k x^{k-2} d y, \quad k=1,3,5 .
$$

Since the orientation along $\gamma_{h}$ is clockwise, we have $I_{k}(h)<0$ for $k=1,3,5$ and $h \in(-1 / 8,0)$. Furthermore to simplify computations, 
we introduce the new variable $z=y+4 x^{2}$, then the equation (2) becomes

$$
\frac{d x}{d t}=z, \quad \frac{d z}{d t}=-x(1-8 z)
$$

and the curve $\gamma_{h}$ has the form

$$
\gamma_{h}=\left\{(x, z) \mid e^{-32 x^{2}+8 z}\left(z-\frac{1}{8}\right)=h, \quad h \in\left(-\frac{1}{8}, 0\right)\right\} .
$$

Thus, along $\gamma_{h}$ we have

$$
\frac{\partial x}{\partial h}=-\frac{1}{64 x h}, \quad \frac{\partial z}{\partial h}=-\frac{\frac{1}{8}-z}{8 z h}
$$

and

$$
z<\frac{1}{8}, \quad \frac{d z}{x}=-(1-8 z) d t<0, \quad d z=-x\left(\frac{1}{z}-8\right) d x .
$$

By Lemma 5 it is obvious that

$$
M^{\prime}(h)=\int_{\gamma_{h}} \frac{F(x)}{x} d y=\int_{\gamma_{h}} \frac{F(x)}{x} d z,
$$

where $F(x)=\alpha_{1}+3 \alpha_{2} x^{2}+5 \alpha_{3} x^{4}$.

In the following we use the new variables $(x, z)$.

Lemma 6. If $\alpha_{3}=0$, then for $h \in(-1 / 8,0)$ the function $M^{\prime}(h)$ has at most one zero, including its multiplicity, where $M(h)$ is the linear combinations of $I_{1}(h), I_{3}(h)$ and $I_{5}(h)$, shown in $(5)$.

Proof. If $\alpha_{3}=\alpha_{2}=0$, then $\alpha_{1} \neq 0$. By (15) and the second equality of (14) we obtain

$$
M^{\prime}(h)=\alpha_{1} \int_{\gamma_{h}} \frac{d z}{x} \neq 0, \quad h \in(-1 / 8,0) .
$$

If $\alpha_{3}=0, \alpha_{2} \neq 0$, we can rewrite (5) as

$$
M(h)=I_{3}(h)+\alpha I_{1}(h) .
$$

By using (10) we have

$$
M^{\prime}(h)=I_{3}^{\prime}(h)+\alpha I_{1}^{\prime}(h)=\int_{\gamma_{h}} \frac{3 x^{2}+\alpha}{x} d z .
$$

If $\alpha \geq 0$, then $M^{\prime}(h)<0$, because $\frac{d z}{x}<0$ along $\gamma_{h}$ by (14).

If $\alpha<0$, we denote $x_{0}$ the positive root of $3 x^{2}+\alpha=0$. Suppose that the intersection points of the curve $\gamma_{h}$ and the axis $\{(x, z) \mid z=0\}$ 
are $\left( \pm x_{M}(h), 0\right)$, the most left and the most right points of $\gamma_{h}$, then by (12) $\gamma_{h}$ has two branches $z=z_{i}(x, h)$ with

$$
z_{1}<0<z_{2}<\frac{1}{8} \quad \text { for } \quad x \in\left(-x_{M}(h), x_{M}(h)\right) .
$$

Note that $\gamma_{h}$ tends to the origin as $h \rightarrow-1 / 8^{+}$, monotonically expands as $h$ increases from $-1 / 8$, and tends to infinity in $\pm x$ direction as $h \rightarrow 0^{-}$.

If $h \in\left(-\frac{1}{8}, h_{0}\right]$, where $h_{0}=H\left(x_{0}, 0\right)$, then $x_{M}(h) \leq x_{0}$, the curve $\gamma_{h}$ is located in the strip $\left\{(x, z) \mid 3 x^{2}+\alpha \leq 0\right\}$, see Figure 2(i). Hence $M^{\prime}(h)>0$, because $\frac{d z}{x}<0$ along $\gamma_{h}$ by $(14)$.

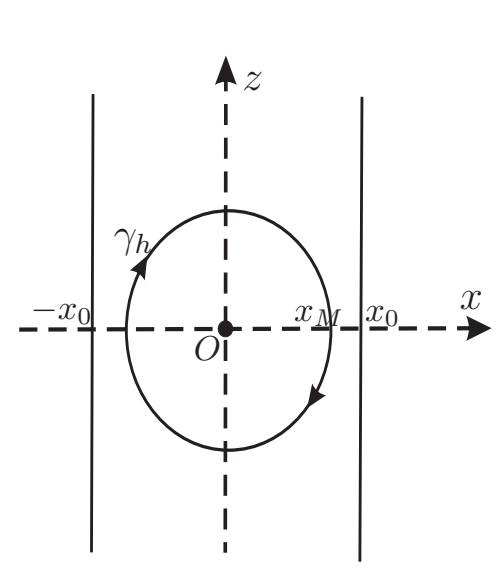

(i) $h \in\left(-\frac{1}{8}, h_{0}\right)$

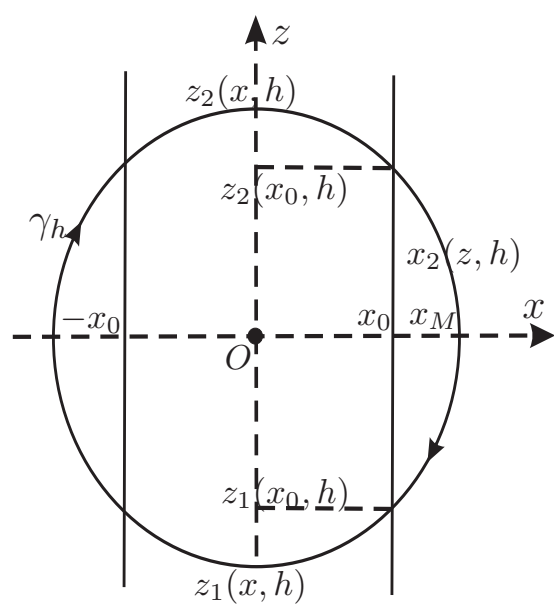

(ii) $h \in\left(h_{0}, 0\right)$

Figure 2. The relative positions of $\gamma_{h}$ and the straight lines $\left\{(x, z) \mid x= \pm x_{0}\right\}$.

If $h \in\left(h_{0}, 0\right)$, then $x_{M}(h)>x_{0}$, the curve $\gamma_{h}$ must cut the straight lines $\left\{(x, z) \mid x= \pm x_{0}\right\}$, see Figure 2(ii). The curve $\gamma_{h}$ is symmetry with respect to $z$-axis, so we will only consider the side $x \geq 0$. We divide the integral form of $\frac{M^{\prime}(h)}{2}$ into two parts as follows:

$$
\frac{M^{\prime}(h)}{2}=\int_{0}^{x_{0}}\left(3 x^{2}+\alpha\right)\left(\frac{1}{z_{1}}-\frac{1}{z_{2}}\right) d x-\int_{z_{1}\left(x_{0}, h\right)}^{z_{2}\left(x_{0}, h\right)} \frac{3 x_{2}^{2}+\alpha}{x_{2}} d z,
$$

where $z_{i}=z_{i}(x, h), i=1,2$; we use the last quality of (14) in the first integral to change $d z$ to $d x$; in the second integral, $x_{2}=x_{2}(z, h)$ is the positive root of $e^{-32 x^{2}+8 z}\left(z-\frac{1}{8}\right)=h$ for any $z \in\left[z_{1}\left(x_{0}, h\right), z_{2}\left(x_{0}, h\right)\right]$, hence $x_{2} \geq x_{0}>0$. 
Making one more derivative with respect to $h$ by using (13), we have

$$
\begin{aligned}
& \frac{M^{\prime \prime}(h)}{2}=\int_{0}^{x_{0}} \frac{3 x^{2}+\alpha}{8 h}\left(\frac{\frac{1}{8}-z_{1}}{z_{1}^{3}}-\frac{\frac{1}{8}-z_{2}}{z_{2}^{3}}\right) d x \\
& \quad-\frac{3 x_{0}^{2}+\alpha}{x_{0}} \cdot\left(\frac{\partial z_{2}\left(x_{0}, h\right)}{\partial h}-\frac{\partial z_{1}\left(x_{0}, h\right)}{\partial h}\right)+\int_{z_{1}\left(x_{0}, h\right)}^{z_{2}\left(x_{0}, h\right)} \frac{3 x_{2}^{2}-\alpha}{64 h x_{2}^{3}} d z<0,
\end{aligned}
$$

because $3 x^{2}+\alpha<0$ for $x \in\left(0, x_{0}\right), z_{1}<0<z_{2}<\frac{1}{8}$ (see (17)), $h<0$, and $3 x_{2}^{2}-\alpha>0$. Besides, $3 x_{0}^{2}+\alpha=0$ and $\left|\frac{\partial z_{2}\left(x_{0}, h\right)}{\partial h}-\frac{\partial z_{1}\left(x_{0}, h\right)}{\partial h}\right|$ is bounded by (13), so the above middle term is zero and we will directly omit the similar terms in further calculations.

Thus, $M^{\prime}(h)$ has at most one zero on $\left(h_{0}, 0\right)$. As we have proved that $M^{\prime}(h)>0$ on $h \in\left(-\frac{1}{8}, h_{0}\right]$, hence $M^{\prime}(h)$ has at most one zero on $\left(-\frac{1}{8}, 0\right)$.

If $\alpha_{3} \neq 0$ in (5), without loss of generality we consider

$$
M(h)=\alpha I_{1}(h)+\beta I_{3}(h)+I_{5}(h), \quad h \in\left(-\frac{1}{8}, 0\right) .
$$

where $\alpha$ and $\beta$ are arbitrary constants. From (10) we have

$$
M^{\prime}(h)=I_{5}^{\prime}(h)+\beta I_{3}^{\prime}(h)+\alpha I_{1}^{\prime}(h)=\int_{\gamma_{h}} \frac{F(x)}{x} d z
$$

where

$$
F(x)=5 x^{4}+3 \beta x^{2}+\alpha .
$$

Lemma 7. If $\beta \geq 0$, then $M^{\prime}(h)$ has at most one zero on $\left(-\frac{1}{8}, 0\right)$, including its multiplicity.

Proof. When $\alpha \geq 0$, it is obvious that $M^{\prime}(h)<0$, because $\frac{d z}{x}<0$ along $\gamma_{h}$. Hence we suppose $\alpha<0$ in the rest part, and denote the only positive root of $F(x)=0$ by $x_{0}$, and $h_{0}=H\left(x_{0}, 0\right)$.

If $h \in\left(-\frac{1}{8}, h_{0}\right]$, then $x_{M}(h) \leq x_{0}$, the curve $\gamma_{h}$ is located in the strip $\{(x, y) \mid F(x)<0\}$. Hence $M^{\prime}(h)>0$.

If $h \in\left(h_{0}, 0\right)$, then $x_{M}(h)>x_{0}$, the proof is completely similar to the proof of Lemma 6 , so we only list some different computations. We first rewrite $M^{\prime}(h)$ as

$$
\frac{M^{\prime}(h)}{2}=\int_{0}^{x_{0}}\left(\frac{F(x)}{z_{1}}-\frac{F(x)}{z_{2}}\right) d x-\int_{z_{1}\left(x_{0}, h\right)}^{z_{2}\left(x_{0}, h\right)} \frac{F\left(x_{2}\right)}{x_{2}} d z .
$$


Then by (13) we obtain

$$
\begin{aligned}
\frac{M^{\prime \prime}(h)}{2}= & \int_{0}^{x_{0}} \frac{F(x)}{8 h}\left(\frac{\frac{1}{8}-z_{1}}{z_{1}^{3}}-\frac{\frac{1}{8}-z_{2}}{z_{2}^{3}}\right) d x \\
& +\int_{z_{1}\left(x_{0}, h\right)}^{z_{2}\left(x_{0}, h\right)} \frac{G\left(x_{2}\right)}{64 h x_{2}^{3}} d z
\end{aligned}
$$

where

$$
G(x)=15 x^{4}+3 \beta x^{2}-\alpha .
$$

Now $\beta \geq 0, \alpha<0$, by using (20) (together with the definition of $x_{0}$ ), (17), (23) and $h<0$ we obtain $M^{\prime \prime}(h)<0$, hence $M^{\prime}(h)$ has at most one zero on $\left(-\frac{1}{8}, 0\right)$.

Now suppose $\beta<0$ and we start from the simple case $\alpha \leq 0$.

Lemma 8. If $\beta<0, \alpha \leq 0$, then $M^{\prime}(h)$ has at most one zero on $\left(-\frac{1}{8}, 0\right)$, including its multiplicity, where $M(h)$ is shown in (18).

Proof. In this case, $F(x)=0$ has exactly one positive root $x_{0}$. Similar to the proof of Lemma 7 , we denote $h_{0}=H\left(x_{0}, 0\right)$, if $h \in\left(-\frac{1}{8}, h_{0}\right]$, then $x_{M}(h) \leq x_{0}, F(x) \leq 0$ along $\gamma_{h}$, hence $M^{\prime}(h)>0$.

If $h \in\left(h_{0}, 0\right)$, then $x_{M}(h)>x_{0}$, we get the same expressions (21) and (22). Now $\beta<0, \alpha \leq 0$, hence $F(x)<0$ on $\left(0, x_{0}\right)$; when $x \geq$ $x_{0}$, we have $F(x) \geq 0$, then $G(x)=3 F(x)-6 \beta x^{2}-4 \alpha>0$, hence $M^{\prime \prime}(h)<0$. In any case we obtain that $M^{\prime}(h)$ has at most one zero for $h \in(-1 / 8,0)$.

Finally we consider the most complicated case $\beta<0$ and $\alpha>0$.

Lemma 9. If $\beta<0$ and $\alpha>0$, then $M^{\prime}(h)$ has at most two zeros on $\left(-\frac{1}{8}, 0\right)$, including their multiplicities, where $M(h)$ is shown in (18).

Proof. Since $\beta<0$ and $\alpha>0, F(x)=0$ may have no positive root, a double positive root, or two different positive roots. In the first two cases, it is obviously $M^{\prime}(h)<0$ for all $h \in(-1 / 8,0)$, because $F(x)>0$ for all $x$, except for the possible double root of $F$, and $\frac{d z}{x}<0$ along $\gamma_{h}$.

It remains to consider the case that $F(x)=0$ has two different positive roots. In this case we denote the smaller root by $x_{0}$, then

$$
x_{0}^{2}=\frac{-3 \beta-\sqrt{9 \beta^{2}-20 \alpha}}{10}, \quad 9 \beta^{2}-20 \alpha>0 .
$$

We will prove two assertions, and denote $h_{0}=H\left(x_{0}, 0\right)$. 
Assertion 1: $M^{\prime}(h)<0$ for $h \in\left(-\frac{1}{8}, h_{0}\right]$.

Since $x_{M}(h) \leq x_{0}$ for $h \in\left(-\frac{1}{8}, h_{0}\right]$, the curve $\gamma_{h}$ is located in the strip $\left\{(x, z)|| x \mid \leq x_{0}\right\}$. Notice $F(x)>0$ on $\left(0, x_{0}\right)$, because $\alpha>0$. Similar to the proof of Lemma 7, we can get $M^{\prime}(h)<0$. This assertion is proved.

Assertion 2: $M^{\prime}(h)$ has at most two zeros (including the multiplicities) for $h \in\left(h_{0}, 0\right)$.

In this case $x_{M}(h)>x_{0}$. We get the same forms (21) and (22). It is easy to see from (23) that $G(x)$ has exactly one positive root for $\beta<0, \alpha>0$, and it is a simple root. Besides, $G(0)=-\alpha<0$, and by using (23) and (24) we have

$$
G\left(x_{0}\right)=20 x_{0}^{4}+6 \beta x_{0}^{2}=-2 x_{0}^{2} \sqrt{9 \beta^{2}-20 \alpha}<0 .
$$

Hence the unique positive root of $G(x)$, denoted by $x_{1}$, satisfies $x_{1}>$ $x_{0}>0$.

If $h \in\left(h_{0}, h_{1}\right]$, then $x_{0}<x_{M}(h) \leq x_{1}$. Obviously $G(x)<0$ on $\left(x_{0}, x_{1}\right)$. From $(22)$ we have $M^{\prime \prime}(h)>0$, because $F(x)>0$ on $\left(0, x_{0}\right)$, $h<0, z_{1}<0<z_{2}<\frac{1}{8}$, and $x_{2}>0$.

If $h \in\left(h_{1}, 0\right)$, then $x_{M}(h)>x_{1}$, we divide the second integral in (22) into two parts, use the last equality of (14) in the first part, and move $(-h)$ to the left side in the whole equality, we have

$$
\begin{aligned}
& \frac{(-h) M^{\prime \prime}(h)}{2}=\int_{0}^{x_{0}} \frac{F(x)}{8}\left(\frac{\frac{1}{8}-z_{2}}{z_{2}^{3}}-\frac{\frac{1}{8}-z_{1}}{z_{1}^{3}}\right) d x \\
& \quad-\int_{x_{0}}^{x_{1}} \frac{G(x)}{64 x^{2}}\left(\frac{1}{z_{2}}-\frac{1}{z_{1}}\right) d x-\int_{z_{1}\left(x_{1}, h\right)}^{z_{2}\left(x_{1}, h\right)} \frac{G\left(x_{2}\right)}{64 x_{2}^{3}} d z .
\end{aligned}
$$

Making one more derivative with respect to $h$ by (13), we obtain

$$
\begin{aligned}
& \frac{\left[(-h) M^{\prime \prime}(h)\right]^{\prime}}{2} \\
& =\int_{0}^{x_{0}} \frac{F(x)}{32 h}\left(\frac{\left(\frac{1}{8}-z_{2}\right)\left(\frac{3}{16}-z_{2}\right)}{z_{2}^{5}}-\frac{\left(\frac{1}{8}-z_{1}\right)\left(\frac{3}{16}-z_{1}\right)}{z_{1}^{5}}\right) d x \\
& \quad-\int_{x_{0}}^{x_{1}} \frac{G(x)}{8^{3} h x^{2}}\left(\frac{\frac{1}{8}-z_{2}}{z_{2}^{3}}-\frac{\frac{1}{8}-z_{1}}{z_{1}^{3}}\right) d x \\
& \quad+\int_{z_{1}\left(x_{1}, h\right)}^{z_{2}\left(x_{1}, h\right)} \frac{15 x_{2}^{4}-3 \beta x_{2}^{2}+3 \alpha}{64^{2} h x_{2}^{5}} d z<0,
\end{aligned}
$$


because $F(x)>0$ on $\left(0, x_{0}\right), h<0, z_{1}<0<z_{2}<\frac{1}{8}, G(x)<0$ on $\left(x_{0}, x_{1}\right), x_{2}>0$, and $15 x_{2}^{4}-3 \beta x_{2}^{2}+3 \alpha>0$ for $\beta<0$ and $\alpha>0$.

Thus, $(-h) M^{\prime \prime}(h)$, hence $M^{\prime \prime}(h)$, has at most one zero on $\left(h_{1}, 0\right)$. Since we have proved that $M^{\prime \prime}(h)>0$ on $\left(h_{0}, h_{1}\right]$, we get that $M^{\prime \prime}(h)$ has at most one zero on $\left(h_{0}, 0\right)$, hence $M^{\prime}(h)$ has at most two zeros on $\left(h_{0}, 0\right)$. the Assertion 2 is proved.

Supping up the results in Assertion 1 and Assertion 2, we obtain that $M^{\prime}(h)$ has at most two zeros on $\left(-\frac{1}{8}, 0\right)$. All multiplicities of the zeros are taken into account.

Proof of Theorem 1. We claim that $M(h)$ has at most two zeros on $\left(-\frac{1}{8}, 0\right)$, including their multiplicities. Otherwise, if $M(h)$ has at least three zeros on $\left(-\frac{1}{8}, 0\right)$, then since $M\left(-\frac{1}{8}\right)=0, M^{\prime}(h)$ would have at least three zeros on $\left(-\frac{1}{8}, 0\right)$, which contradicts Lemmas 6-9.

\section{ACKNOWLEDGEMEnTS}

The first author is partially supported by NNSF of China grant number 11771315, The second author is partially supported by NNSF of China grant number 11771282 . The third author is supported by the Ministerio de Economía, Industria y Competitividad, Agencia Estatal de Investigación grant MTM2016-77278-P (FEDER), the Agència de Gestió d'Ajuts Universitaris i de Recerca grant 2017SGR1617, and the H2020 European Research Council grant MSCA-RISE-2017-777911.

The authors would like to thank the reviewer for his/her valuable suggestion that improved the representation of the paper.

\section{REFERENCES}

[1] V. I. Arnold, Loss of stability of self-oscillations close to resonance and versal deformations of equivariant vector fields, Funct. Anal. Appl. 11 (1977), 85-92.

[2] V. I. Arnold, Ten problems, Adv. Soviet Math. 1 (1990), 1-8.

[3] C. Christopher and C. Li, Limit cycles in differential equations, Birkhäuser, Boston, 2007.

[4] F. Dumortier, J. Llibre and J. C. Artés, Qualitative theory of planar differential systems, Universitext, Spring-Verlag, 2006.

[5] F. Dumortier and R. Roussarie, Birth of canard cycles, Discrete Contin. Dyn. Syst. 2 (2009) 723-781.

[6] J. Figuerasa, W. Tucker and J. Villadelprat, Computer-assisted techniques for the verification of the Chebyshev property of Abelian integrals, J. Differential Equations 254 (2013), 3647-3663.

[7] I. D. Iliev, Perturbation of quadratic centers, Bull. Sci. math. 122 (1998), 107-161. 
${ }^{1}$ School of Mathematics (Zhuhai), Sun Yat-Sen University, 519086, Zhuhai, China

E-mail address: liuchangj@mail.sysu.edu.cn

${ }^{2}$ School of Mathematical Sciences, Peking University, BeiJing 100871 , CHINA

E-mail address: licz@math.pku.edu.cn

${ }^{3}$ Departament de Matemàtiques, Universitat Autònoma de Barcelona, 08193 Bellaterra, Barcelona, Catalonia, Spain

E-mail address: jllibre@mat.uab.cat 